\title{
Accounting for taxonomic distance in accuracy assessment of soil class predictions
}

\author{
David G. Rossiter ${ }^{\mathrm{a}, \mathrm{c}, \mathrm{d}, *}$, Rong Zeng ${ }^{\mathrm{a}, \mathrm{b}}$, Gan-Lin Zhang ${ }^{\mathrm{a}, \mathrm{b}}$ \\ ${ }^{a}$ State Key Laboratory of Soil and Sustainable Agriculture, Institute of Soil Science, \\ Chinese Academy of Sciences, Nanjing, 210008, PR China \\ ${ }^{b}$ University of the Chinese Academy of Sciences, Beijing, 100049, PR China \\ ${ }^{c}$ ISRIC World Soil Information, PO Box 353, 6700 AJ Wageningen, the Netherlands \\ ${ }^{d}$ Section of Soil 83 Crop Sciences, Cornell University, 242 Emerson Hall, Ithaca NY \\ 14850, USA
}

\begin{abstract}
Evaluating the accuracy of allocation to classes in monothetic hierarchical soil classification systems, including the World Reference Base for Soil Classification, US Soil Taxonomy, and Chinese Soil Taxonomy, is poorly-served by binomial methods (correct/incorrect allocation per evaluation observation), since some errors are more serious than others in terms of soil properties, map use, pedogenesis, and ease of mapping. Instead, evaluations should account for the taxonomic distance between classes, expressed as class similarities, giving partial credit to some incorrect allocations. These can then be used in weighted accuracy measures, either direct measures of agreement or measures that account for chance agreement, such as the tau index. Similarities can be determined in one of four ways: (1) by the expert opinion of a soil classification specialist; (2) by the distance between classes in a numerical taxonomy assessment; (3) by distance within a taxonomic hierarchy; or (4) by an error loss function. Expert opinion can be from the point of view of the map user, to assess map utility, or map producer, to assess mapping skill. Examples are given of determining similarity be-


tween a subset of Chinese Soil Taxonomy classes by expert opinion and by numerical taxonomy from soil spectra, and then using these for weighted accuracy assessment. A method for assessing the accuracy of probabilistic predictions of several classes at a location is also proposed.

Keywords: soil class maps, accuracy assessment, map evaluation

*Corresponding author

Email addresses: david.rossiter@isric.org (David G. Rossiter), dgr2@cornell.edu (David G. Rossiter), rzeng@issas.cas.cn (Rong Zeng), glzhang@issas.cas.cn (Gan-Lin Zhang)

Preprint submitted to Geoderma

January 2, 2017 
1 1. Introduction

2 Soil classes are information carriers that present a holistic view of groups 3 of soil individuals with a definite "personality". They have proven useful

4 as the units of soil survey interpretation and to explain soil geography, 5 for example using the World Reference Base for Soil Classification (WRB) 6 (Driessen et al., 2001; FAO, 2014; Van Wambeke and Nachtergaele, 2003), 7 US Soil Taxonomy (ST) (Buol et al., 2011; Soil Survey Staff, 2014), or Chi8 nese Soil Taxonomy (CST) (Cooperative Research Group on Chinese Soil 9 Taxonomy, 2001; Gerasimova, 2010). These are all monothetic hierarchical 10 classification systems, where individuals are allocated to single classes ac11 cording to sharp thresholds, so that all members of any class share a certain 12 set of features that are not present in any members of other classes (Sneath 13 and Sokal, 1973). This allows the construction of hierarchical keys by which 14 a soil individual is allocated to exactly one class.

15 Any predictive method, for example digital (McBratney et al., 2003) or 16 predictive (Scull et al., 2003) soil mapping (DSM, PSM) that allocates indi17 viduals to single monothetic classes is sure to produce incorrect allocations 18 with respect the true state of nature. The producer of such a prediction is 19 obliged to evaluate and report its accuracy, so that the map user can de20 termine whether the map is fit for an intended use, and so that the skill of 21 the producer may be assessed. There is an extensive literature on measures 22 of classification accuracy (e.g., Stehman and Czaplewski, 1998). These all 23 depend on a set of evaluation observations (often called validation obser24 vations, but see Oreskes et al. (1994) for the use of 'evaluation' in place of 25 'validation'): the true state of nature is compared to the prediction. In the case of allocation to soil classes, the "true" state is presumed to be the allo- 
cation assigned by the professional classifier. These observations are usually presented as a cross-classification matrix (also called a confusion matrix): rows represent classes as predicted, columns the reference class as presumed correct, and cell entries are the number of evaluation observations for each combination.

Most evaluations of classification accuracy consider all misallocations, represented by the off-diagonal entries of the cross-classification matrix, to be equally serious errors. However, when the predictions are of soil classes, it is obvious that not all errors are equally serious. In the first place, professional classifiers often disagree on the correct allocation in doubtful, and sometimes not so doubtful, cases, as anyone who has been on a soil correlation exercise can attest. Certain diagnostic features are subjective, despite the efforts of the creators of the keys to make them as objective as possible. For example, the Soil Taxonomy definition of a fragipan requires, in part that "the layer shows evidence of pedogenesis within the horizon, or at a minimum, on the faces of structural units" (Soil Survey Staff, 2014, p. 13). This clearly relies on the classifier's opinion of what constitutes "evidence of pedogenesis".

45 Second, the selection of the soil individual, typically a soil profile, for evaluation is subjective, and moving a few meters one way or the other can easily change the classification. For example, in the WRB a mollic horizon, not directly over certain indurated materials, must have a thickness of $20 \mathrm{~cm}$ or more. It is easy to find natural soil bodies shown as delineations on an area-class map where the thickness of a horizon that would otherwise qualify as mollic ranges from 18 to $22 \mathrm{~cm}$. Thus the evaluation observation with a calcic horizon and a dark, organic-rich, high-base saturation epipedon 
53 would be classified as a Kastanozem if the mollic horizon is recognized, but 54 a Calcisol otherwise. Clearly any misallocation by the predictive method can 55 not be expected to do better than the field scientist selecting a supposedly 56 representative individual. An extreme case was reported by Edmonds and 57 Lentner (1986), who found profiles allocated to four Soil Taxonomy soil ${ }_{58}$ orders within $7 \mathrm{~m}$ of each other in a steep hillside map unit in the Ridge 59 and Valley province in Virginia (USA), all with similar use potential and 60 pedogenesis.

61 Third, and most importantly, the consequences of a misallocation for 62 someone using the prediction can differ widely depending on the relation 63 between the allocated class and the actual class. For example, in the USA 64 if a map delineation is allocated to a soil series which is considered a so65 called hydric soil, a large number of restrictions are placed on the land 66 use (Vepraskas, 2015), so predicting a hydric soil where the soil is in fact 67 not hydric imposes a large burden on the land user. The reverse error, ${ }_{68}$ i.e., predicting a non-hydric soil where the soil is in fact hydric, can lead 69 to severe environmental problems such as agricultural chemicals in surface 70 waters. On the other hand, if a delineation is allocated to a moderately well71 drained member of a drainage sequence, when in fact the soil is dominantly 72 well-drained, there is only a slight difference in use potential.

73 Our objective is thus to measure allocation accuracy, taking into account 74 that some errors are more serious than others. This can be from the user's 75 point of view: what is the utility of the predictions to the user who would 76 take the prediction as correct in their decision-making? It can also be from 77 the producer's point of view: how well did they do their job? In both 78 cases the question is how close or how far, in some sense to be defined, 
are the correct and incorrect allocations to each other? In this paper we formalize this concept of taxonomic distance, which we define as the amount of separation between classes in some feature space. We then discuss ways to express and measure this distance, and its inverse, taxonomic similarity.

The concept of taxonomic distance has been used in several studies to compare classifications (Láng et al., 2013; van Huyssteen et al., 2014), to test the structure of existing classification systems (Michéli et al., 2016), as aid to mapping soil classes (Taghizadeh-Mehrjardi et al., 2015), to compare taxonomic and landscape concepts of soil classes (Young and Hammer, 2000), to improve estimates of pedodiversity (McBratney and Minasny, 2007), and as an objective basis for classification (Carré and Jacobson, 2009; Láng et al., 2016; Moore et al., 1972; Odgers et al., 2011). Phillips (2013) used taxonomic adjacency, defined as classes that differ in only one criterion defined by an arbitrary threshold value, to evaluate map unit complexity. We could find only one published study, that of Dobos et al. (2014), and one unpublished BSc thesis, that of van Beek (2014), which use taxonomic distance to evaluate mapping accuracy. The work of Dobos et al. (2014) was by their own conclusion only a preliminary proof of concept; the work of van Beek (2014) is explained in $\S 3.2$, below.

Therefore the objectives of this paper are to: (1) explain how the conventional classification accuracy assessment method using the cross-classification matrix can be adjusted to account for taxonomic distance; (2) present and discuss different methods for computing taxonomic distance to be used in accuracy assessment. We do not explore the very important issue of how the observations used to build the cross-classification matrix were obtained, i.e., the sampling plan for map evaluation (e.g., Brus et al., 2011; Stehman and 
Czaplewski, 1998). Our objective here is simply to see how adjustments to the conventional accuracy measures can be made, taking into account taxonomic distance between classes.

\section{The ordinary and weighted cross-classification matrices}

The cross-classification matrix $\mathbf{X}$ is the fundamental data structure in accuracy assessment (Congalton and Green, 1999). It is constructed as follows. Suppose that we have $n$ soil profiles and that they have been allocated to $r$ classes, which we denote $i, i=1,2, \ldots, r$. We set up a square asymmetric $r \times r$ matrix $\mathbf{X}$, each row and each column of which corresponds to one class, in the same order. In each cell $(i, j)$ we enter the number of profiles which actually are of class $j$ that have been predicted to belong to class $i$. Thus the diagonals $(1,1),(2,2) \ldots(r, r)$ represent agreement between predicted and actual, and off-diagonals represent different misallocations. The asymmetry arises because there is no reason to expect that a mis-allocation of a profile actually of class $j$ to class $i$ will happen as frequently as the inverse misallocation

From this matrix we compute row sums $x_{i+}$, i.e., the total number allocated to class $i$, and the column sums $x_{+j}$, i.e., the total actually in class $j$. The row-wise proportion of correct allocations $C_{i}=x_{i i} / x_{i+}$ is commonly referred to as the user's accuracy for class $i$; in the case of map accuracy assessment, following Brus et al. (2011) perhaps better termed map unit purity. The errors in this sense $\bar{C}_{i}=1-C_{i}$ are called the errors of commission, i.e., the allocator committed an error in allocating an observation to class $i$.. The column-wise proportion of correct allocations $O_{j}=x_{j j} / x_{+j}$ is commonly referred to as the producer's reliability for class $j$; in the case of 
map accuracy assessment, again following Brus et al. (2011) perhaps better termed soil class representation. The errors in this sense $\bar{O}_{j}=1-O_{j}$ are called the errors of omission, i.e., the allocator did not correctly allocate an observation to class $j$. The overall accuracy is $A_{o}=\sum_{i=1}^{r} x_{i i} / n$ is the proportion of all observations correctly allocated.

Our aim is to weight the cross-classification matrix when computing various accuracy statistics. Our method requires that the analyst make a second matrix $\mathbf{W}$, with the same $r \times r$ dimensions as the cross-classification matrix $\mathbf{X}$, containing the weights $w_{i j}$ corresponding to each cell in the crossclassification matrix. The diagonals $w_{i i} \equiv 1$, giving full credit to correct allocations. The off-diagonals usually range from 0 , indicating that there is no credit for this mistaken allocation, to 1 , indicating that the mistake is of no consequence, i.e., that the two classes are considered identical for the purpose of accuracy assessment. It is also possible to assign a negative weight, as low as -1 , to a particularly egregious misclassification in the "expert opinion" method of assigning weights explained in $\S 3.1$, although this option should rarely be used. The weights matrix need not be symmetric, since an error of commission may be considered more or less serious than an error of omission, depending on the point of view (producer vs. user). Thus the weights matrix can be thought of as assigning partial credit, or even a demerit, for each cross-classification error.

The weighted accuracy statistics are then computed as overall agreement $A_{o w}=\sum_{i=1}^{r} \sum_{j=1}^{r} w_{i j} \cdot p_{i j}$, per-class user's accuracy (map unit purity) $C_{i w}=\left(1 / p_{i+}\right) \cdot \sum_{j=1}^{r} w_{i j} \cdot p_{i j}$, and per-class producer's accuracy (soil class representation) $O_{j_{w}}=\left(1 / p_{+j}\right) \cdot \sum_{i=1}^{r} w_{i j} \cdot p_{i j}$, where $i$ and $j$ are the predicted and reference classes, and $p_{i j}$ is the proportion of observations in $\mathbf{X}$ 
predicted in class $i$ but actually in class $j$, i.e., $x_{i j} / n$.

\section{Operational definitions of taxonomic distance}

The key issue is how to create the weights matrix. Finke (2007) proposed that "[w]eighting can be applied to account for errors that are considered less important, for example because the two classes involved are considered to be taxonomically adjacent." However, he then says "[t]here is no agreement on how such taxonomic weighting should be done." We aim to address this issue. Here is where the specifics of soil classification and soil map use become relevant; a similar discussion for land use/land cover weighted accuracy assessment would have other considerations than those presented here. We present four alternatives; they are then compared in the Discussion of $\S 7$.

\subsection{Expert opinion on taxonomic similarity}

The first method for creating the weights matrix is expert opinion on how similar are each pair of classes $(i, j)$ when the allocation is to class $i$ but the observation is actually in class $j$. This rating can be thought of as the expert's opinion of the partial credit to be given to the allocator who makes a certain error, either of omission or commission. This is not necessarily symmetric, nor transitive, i.e., there is no need to check for consistency. It is strictly a pairwise assessment, filled in column-wise: the reference class (column) mapped as the class given in the row. For example the partial credit for allocating an observation that is really of reference class 2 to class 1 is entered in cell $(1,2)=$ (allocated, reference). The asymmetry could come from the error in interpretations due to misallocation, or the 
classifier's judgement about how similar the soils are overall, or how likely it might be to make a small change in the properties and follow another branch of the key at any level. The credit can be from the user's or producer's point of view, according to whether the partial credit should reflect the utility of the map to the user or the skill of the mapper.

As an example from the WRB, consider allocating a deep soil mainly of coarse fragments, fine earth fraction sandy loam, with a cambic horizon ("a subsurface horizon showing evidence of pedogenetic alteration that ranges from weak to relatively strong"). If the mapper estimates that the requirement " $<20 \%$ (by volume) fine earth, averaged over a depth of $75 \mathrm{~cm}$ from the soil surface" from the definition of Leptosols is met, this would be allocated to the Cambic Skeletic Leptosols. Suppose that the volume of fine earth is in fact somewhat higher, $20-60 \%$ (40-80\% coarse fragments), so it does not quality as Leptosol (5th in key order). It will fall through the keys to the Cambisols (29th in key order) and be classified as a Skeletic Cambisol, meeting the qualifier requirement for Skeletic: "having $\geq 40 \%$ (by volume) coarse fragments averaged over a depth of $100 \mathrm{~cm}$ from the soil surface". This is an easy misallocation to make, especially because estimating coarse-fragment volume in the field is notoriously unreliable ${ }^{1}$. So the expert may rate this misallocation as a minor error (1) for interpretations (soil functions), (2) for pedogenesis, (3) for ease of making the mistake in the keys due to imprecise and inconsistent measurement of diagnostic properties, and may assign a partial credit of 0.9 to a predicted Cambic Skeletic

\footnotetext{
${ }^{1}$ This could be determined on a weight basis and converted using particle density for coarse fragments and overall soil bulk density, but the latter would be difficult to measure with so many coarse fragments
} 
Leptosol which is in fact a Skeletic Cambisol. The other direction may receive the same partial credit, if similarity is based on concepts of pedogenesis, but may well be less (say, 0.8) since the use potential of Cambic Skeletic Leptosol is poorer than most Skeletic Cambisols, due to the higher proportion of coarse fragments.

This approach is unwieldy for many classes. In a recent study $^{2}$ soil observations were allocated to 8 orders, 12 suborders, 23 groups and 49 subgroups of CST. These would require $\left(8^{*} 7\right)=56$ (asymmetric) or $\left(8^{*} 7 / 2\right)=28$ (symmetric) comparisons just at the order level; for subgroups this would be $(49 * 48)=2352$ or $(49 * 48 / 2)=1176$ comparisons. However, in practice not all pairs of classes are confused. For any pairs where the cross-classification matrix has a zero, there is no need to assign weight, since it will not affect computations.

The obvious criticism of this approach is its subjectivity. This can be partially offset by consulting several experts and (1) agreeing on the criteria for similarity (use potential, pedogenesis, ease of misallocation or a combination of these), (2) asking the experts to independently assign weights; (3) comparing these for consistency. If consistent, there is no problem; if not, either average the expert opinions or ask the experts to discuss their reasoning and reach a consensus. An advantage of this approach is that the result is per se acceptable to the experts.

${ }^{2} \mathrm{R}$. Zeng et al. "Numerical classification based on whole-profile spectra and soil properties as a complement to hierarchical soil classification", submitted to Geoderma 06-December-2016 


\subsection{Distance between classes in a numerical taxonomy assessment}

The second method for creating the weights matrix is to use numerical taxonomy to establish taxonomic distances between entities as a symmetric distance matrix. Classes may be compared by computing the distances between single representatives of each class, e.g., a modal profile, whether an actual profile on the landscape or a synthetic central concept. They may also be compared from sets of individuals representing each class, in which case the distances may be computed as the average of individual Euclidean or other single-linkage distances between the several representatives of each class, or as Mahalanobis distances between these, which take into account the intra-class variances as well as the distances between classes.

The advantage of using numerical taxonomy is that similarities are based on measured soil properties. Numerical taxonomists working in biological systematics (e.g., Sneath, 1995; Sneath and Sokal, 1973) advise selecting a large number of properties to avoid bias, despite the obvious problems of intercorrelation and previous selection of properties to be measured (Smith, 1986, §1.22.7). We illustrate this with visible-near infrared (VNIR) spectra in $§ 5.2$, below.

Another approach is to select those properties considered relevant for the purpose. For example, Bidwell and Hole (1964) in their study of Kansas soils selected properties that had been used in the then-current USA classification system, so that the computed distances were directly interpretable in terms of that system. A similar approach was taken by Minasny et al. (2010) in their study of global pedodiversity, where they selected indicators used in the studied classification system, in their case the WRB.

A particular problem with soil profiles is how to compare profiles of 13 
different thicknesses. Various solutions are compared by Beaudette et al. (2013); we explain one that we adopted in $§ 5.2$, below. Thus the apparent objectivity of numerical taxonomy is subject to intervention by the analyst; however this can be an advantage if the properties are selected specifically for the purposes of similarity according to some criteria of interest, and the selection criteria are transparent to the user of the accuracy statistics.

There are a wide variety of distance measures that can be used. These are discussed thoroughly in many sources (e.g., de Gruijter, 1977; Sneath and Sokal, 1973). A common choice is that of Gower (1971), in which similarity between objects $i$ and $j$ is defined as the average score taken over all possible $k$ attribute comparisons: $S_{i j}=\sum_{k=1}^{v} s_{i j k} / \sum_{k=1}^{v} \delta_{i j k}$, where $\delta_{i j k}$ is an indicator which shows whether attribute $k$ is present in both objects and thus can be compared. The per-attribute similarities $s_{i j k}$ for each of the $v$ possible attributes may be computed for dichotomous (e.g., present or absent), qualitative (classes), or quantitative variables. In the most common case of a quantitative variable, e.g., a spectral reflectance in a single band, similarity is computed as $s_{i j k}=1-\left(\left|x_{i}-x_{j}\right| / R_{k}\right)$, where $R_{k}$ is the range of the variable within the sample or the population from which the sample was taken.

Taxonomic distances may be based on a set of indicators that are assumed to adequately characterize each class, and to have equal weight for that purpose. This was proposed by Minasny et al. (2010) in their study of global pedodiversity, and applied to the 30 reference groups of the WRB. They coded the presence of 21 diagnostic soil and environmental conditions as the indicators "present" (1) or "absent" (0) in the definition of the reference group. The taxonomic distance between classes $i$ and $j$ was then 

${ }_{299} S=1-(\mathbf{D} / \max \{\mathbf{D}\})$.

computed as $d_{i j}=\sqrt{\left(\mathbf{x}_{i}-\mathbf{x}_{j}\right)^{T}\left(\mathbf{x}_{i}-\mathbf{x}_{j}\right)}$, where $\mathbf{x}$ is the vector of indicators, either 0 or 1 . Lower values of this measure are more similar, i.e., more common 0's and 1's. For example, Solonetz and Solonchaks agree in 14 of the 21 indicators, so their distance is $\sqrt{7} \approx 2.65$; perhaps surprisingly Solonetz and Ferralsols agree in 17 of the indicators, so their distance by this measure is only 2. Van Beek (2014) then used these distances as weights to prepare weighted accuracy measures for three class maps of African soils (HWSD, S-World, and SoilGrids1km), using the Africa Soil Information Service (AfSIS) profile database (Leenaars, 2013) as the reference classes. Each entry of the cross-classification matrix was multiplied by the associated taxonomic distance, as we propose here, and then subtracted from an assumed perfect accuracy: $A_{o w}=1-\sum_{i=1}^{r} \sum_{j=1}^{r} p_{i j} \cdot d_{i j}$. The computed accuracy of the three products increased from $0.33,0.27$ and 0.28 to 0.57 , 0.55 , and 0.58 , respectively, i.e., more or less doubled. Our contention is that these weighted accuracies better represent the usefulness of the maps, when their users base decisions or model inputs on the mapped classes.

The distance matrix is expressed on some arbitrary scale depending on the number of properties, their measurement scales and ranges across the set of entities, and the method of accounting for covariance. One method to standardize the distances is to divide each dissimilarity $d$ by the maximum dissimilarity in the set $\max \{\mathbf{D}\}$. The distance matrix measures dissimilarity: higher numbers represent greater differences. To use in weighted accuracy assessment, the matrix must be converted to a similarity matrix 


\subsection{Distance within the taxonomic hierarchy}

The third method for creating the weights matrix is to use distance within a hierarchical system: how far in the hierarchy one must go to match classifications. For example, a Soil Taxonomy Fragiudept and a Dystrudept differ in the presence of a fragipan, and the designers of Soil Taxonomy decided that this difference is important at the third level of the classification. A misallocation between these could be assigned a similarity of $2 / 3$, since they agree at the second, but not the third, level. In the same way, a difference in the predicted moisture regime at suborder level within the same order, e.g., Udepts vs. Ustepts, would be assigned a similarity of $1 / 2$. A complication is if the name at the target or next lower level implies an intergrade to the property that caused the difference at the target level. For example, the Pawnee series (Oxyaquic Vertic Argiudolls) differs from the Wymore series (Aquertic Argiudolls) at the subgroup level, implying a similarity of $3 / 4$. These are geographically-associated soil series on hillslopes of loess-mantled dissected till plains in Nebraska and Kansas, USA (USDANRCS, N.D.). However, 'aquertic' implies 'vertic', and 'oxyaquic' is just one drainage class above 'aquic' (as used in Aquertic). Thus rather than a similarity of 3/4 (in different subgroups of the Arigudolls great group) perhaps the similarity is more like $5 / 6$. This kind of refinement would have to be established by expert rules, for example identifying related intergrades expressed as subgroup modifiers.

This method assumes that the hierarchical levels represent degrees of similarity, and this was certainly the aim of the designers of hierarchical systems. This may be true for the lower levels, e.g., families and subgroups, but has less justification at higher categories, because these are so broad as 
to include individuals that are intergrades to adjacent classes. In the example of the Udepts vs. Ustepts, in transitional regions between these moisture regimes the use potential of these soils are quite similar, whereas at the extremes of these regimes (transition to aridic and perudic regimes, respectively) the use potential of the soils are quite different. Further, this method does not account for similarity of concepts at the same level. Fragiudepts have a fragipan, which has a large influence on land use, so the contrast with a Dystrudept is fairly strong. Another contrast at the same taxonomic level, Humudepts (with mollic or umbric epipedon) and Eutrudepts (high base status) seems much weaker, as both have indications of high natural soil fertility. Another contrast is even stronger: Sulfudepts vs. Eutrudepts. Rating all these contrasts as $2 / 3$ the same similarity does not capture the functional distance between them, nor the ease of misallocation.

To apply this method to WRB 2014 (FAO, 2014) the principal, secondary, and supplementary qualifiers of the second level (below the reference groups) could be considered as three levels. However, all of these can be multiple, and rules would have to be developed to assign a difference within one of the qualifier groups when classes have different numbers of qualifiers.

\subsection{Error loss function}

The fourth method for creating the weights matrix is to use a loss function which is or can be converted to a monetary value. This is appropriate if the allocation will be used to make class-based management decisions. In this case the loss due to a misallocation can serve as the weighting: the more loss, the less credit that can be given for a misallocation. This does 
not have to be symmetric.

As an example, consider the site index (Avery and Burkhart, 2015, §15) for an adapted forest species, one of the interpretations provided by the US Natural Resources Conservation Service (NRCS) for each USA soil series. This is supposed to be a linear measure of the growth of the species over time, leading to the economically useful timber volume per unit land area, and is established by calibration plots of the species. If the soil map is used to locate suitable planting sites for a species, there would be an economic loss in planting in less-than-ideal sites, which should correspond to the difference between site indices of the two classes. This could be asymmetric, for example to give partial credit for mapping a less-productive soil, but not vice-versa. The user will get an unexpected bonus if the site turns out to be better than mapped, but will make a decision to plant or not based on the map.

An example from the middle coastal plain of North Carolina illustrates this method. Loblolly pine (Pinus taeda) is an important softwood timber species of the southern USA. In its range of adaptation this species produces best on poorly-drained upland sites with thick argic horizons. Table 1 shows the soil series along a drainage sequence from poorly (Rains) to excessively (Wagram) well-drained series in related families of Ultisols, as well as a a geographically-associated series (Tarboro) of Psamments on a lower geomorphic surface, well-drained and with a sandy profile throughout, thus more prone to drought. Site indices and classification in Soil Taxonomy per the Official Series Descriptions were taken from Web Soil Survey (USDA-NRCS, 2013). 


\footnotetext{
${ }^{3}$ The discrepancy where Goldsboro is rated higher than Lynchburg is unexplained and may represent a recording error.
} 
result in complete loss, similarity 0.

\section{Computation}

Once the cross-classification matrix (from the evaluation sample) and the weights matrix (as outlined above) are created, accuracy statistics can be computed as presented in $\S 2$. The obvious statistics for a map or allocation user are naïve statistics, a direct measure of the agreement between predicted and reference classes. The user's statistics from these are the accuracy that the user can expect, and the producer's are measures of how successfully the producer identified the correct class. However, if the taxonomic distances are based on the differences in utility to the map user, the weighted accuracy statistics give a more realistic indication of the map accuracy. This is certainly the case if an error loss function or numerical taxonomy was used to compute the distances, and also if expert opinion on similarity from the point of view of map interpretation was used.

If the accuracy assessment is to evaluate the skill of the allocator or mapper, many studies use Cohen's kappa (Cohen, 1960) for the producer's statistics and overall accuracy. This modifies the naïve or weighted statistics to adjust for chance agreement, and is especially relevant if the reference data has only a few classes or if some classes occur much more frequently than others. It suffers from a major conceptual flaw, namely the assumption that the reference and allocations are independent and that both the user's and producer's marginal class probabilities are known a priori before the allocation. These assumptions are needed to compute the effect of a random allocation of observations to classes, according to the known conditional probabilities. These may be satisfied in the case of independent observers 


$$
\tau=\frac{\theta_{1}-\theta_{2}}{1-\theta_{2}}, \text { where } \theta_{1}=\sum_{i=1}^{r} p_{i i}, \theta_{2}=\sum_{i=1}^{r} p_{i} \cdot p_{+i}
$$

${ }_{449}$ Note the use of the a priori probabilities of class membership $p_{i}$, instead 
of the post-allocation row proportions $p_{i+}$ as in kappa, to represent the row proportions. These are assigned by the analyst, with the obvious restriction that $\sum_{i} p_{i}=1, \forall i$. The first coefficient, $\theta_{1}$, is the overall naïve accuracy $A_{o}$. The second, $\theta_{2}$, is the expected overall accuracy if allocations were made randomly to reference classes, taking into account prior probabilities of each reference class. So $\tau$ is the ratio between the actual proportional errors to the proportion of errors expected by chance allocation, taking into account the prior probabilities of allocation. This can even be less than 0 for a worse-than-random allocation.

As with the naïve statistics, tau can be weighted, symbolized as $\tau_{w}$. This is computed as:

$$
\theta_{w 1}=\sum_{i=1}^{r} \sum_{j=1}^{r} w_{i j} \cdot p_{i j}, \theta_{w 2}=\sum_{i=1}^{r} \sum_{j=1}^{r} w_{i j} \cdot p_{i} \cdot p_{+j}
$$

and these modified coefficients substituted into Equation 1.

The revised coefficient $\theta_{w 1}$ (overall accuracy) includes the partial credit for errors; if all the off-diagonals of the weights matrix are zero, this reduces to $\theta_{1}$. Similarly, $\theta_{w 2}$ (chance agreement) accounts for partial credit, i.e., increases the chance agreement if any off-diagonal is not zero.

We have implemented the calculation of naïve and tau statistics in the $\mathrm{R}$ function tau.w, which results can be summarized by $\mathrm{R}$ function summary.tau.w. These are provided in file WeightedAccuracy.R as supplementary material.

\section{Examples}

In this section we illustrate weighted accuracy assessment with examples of various kinds of similarity estimates. 


\subsection{Expert opinion}

In the first example we evaluate the taxonomic distance by expert opinion. The cross-classification matrix is from Zeng et al. (2016), who evaluated how well VNIR spectra could distinguish observations from a set of soil classes. We chose this example because it has a small matrix, with which we can easily illustrate the computations. Allocation was to subgroups in Chinese Soil Taxonomy, the subgroups coded as AV (Aquic Vertosols), AC (Aquic Cambosols), UC (Udic Cambosols), UA (Udic Argosols), SA (Stagnic Argosols) and OP (Orthic Primosols). The results of the allocation from the VNIR spectra are shown in Table 2. From the user's perspective, the Aquic Vertosols were never correctly predicted, and Aquic Cambosols predictions were distributed among four incorrect reference classes as well as the correct one. From the producer's perspective, actual Aquic Cambosols were predicted to be in three incorrect reference classes, in addition to the correct one.

Table 3 shows the partial credit assigned to each misallocation, in the expert opinion of an experienced soil classifier from the Nanjing Soil Science Research Institute, Chinese Academy of Sciences. Interestingly, the similarities assigned by the expert are not symmetric. For example, misallocating an actual Aquic Cambosol as a Stagnic Argosol is given the highest partial credit (0.35), while the reverse misallocation only is given a partial credit of 0.30 . 
The resulting accuracy assessment from applying the computation methods of $\S 4$ for both the unweighted and weighted case is shown in Table 4 . Since the automatic classifier did not use any information on the relative proportion of reference classes, the prior probabilities for tau were set as the inverse of the number of classes, i.e., equal prior probabilities. Accounting for partial credit leads to a slight increase in overall accuracy, from 0.763 to 0.804 . User's accuracy increased for all classes, because all classes had errors of commission, and all errors received some partial credit. The largest increase in user's accuracy was for for the Aquic Vertosols: from 0 (nothing correct) to 0.12. This is because the eight misallocations of Aquic Vertosols as Stagnic Argosols (4) or Aquic Cambosols (4) were given a partial credit of 0.1 and 0.15 , respectively. The expert considered the aquic or stagnic moisture regimes to be important control land use, the Argosols to by definition have high clay as do the Vertosols, and that most of the Cambosols in this study area are fairly clayey. However these similarities only resulted in a small amount of partial credit, because the vertic properties were not recognized in the two erroneously-allocated classes. Per-class producer's accuracy increased most for this same class. Here the misallocations were to the Aquic Vertosols (8) from the Stagnic Argolsols (3), Udic Argosols (1) and Aquic Cambosols (4), all with some partial credit. The $\tau$ statistic increased only slightly, from 0.716 to 0.725 . These are not much less than the corresponding naïve statistics, because the classifier mostly identified the large and small classes, and this is an improvement from the equal prior probabilities. 


\subsection{Numerical taxonomy}

In the second example we use similarity as distance in property space as computed by the techniques of numerical taxonomy. We illustrate this with spectral space as the soil properties to be used as an objective quantitative measure; the same technique could be used with a set of field and laboratory quantitative soil properties. Soil spectra are especially suited for numerical taxonomy because of the large amount of quantitative information, which is influenced by many soil properties. This approach to allocation has been used successfully by, among others, Viscarra Rossel and Webster (2011). Our example dataset contains 189 soil profiles from a field campaign in the Heihe River watershed, northwestern China, with a combined 830 genetic horizons (average 4.4 horizons per profile), from the Zeng et al. manuscript mentioned in $\S 4$. Each profile had been classified to CST orders by experts in the CST.

Samples from each field-identified and sampled genetic horizon were scanned with a Cary 5000 spectrometer under controlled laboratory conditions, providing 2151 bands in the visible and near infrared (VNIR) range of 350-2500 nm. The profiles were scaled to one meter thickness, i.e., either expanded or contracted according to the original thickness; their constituent horizons were proportionally scaled within this one meter. The 830 2151band spectra were reduced with standardized principal components analysis (sPCA); four components explained $98 \%$ of the total variance. This shows the large amount of redundancy among spectral bands and between horizons in a given profile. The first four principal components were then stacked into profiles using the slab function of the aqp "Algorithms for Quantitative Pedology" R package (Beaudette et al., 2013) to convert per- 
horizon values to average values for a set of standard depth slices, in this case those specified by the GlobalSoilMap.net project (GlobalSoilMap.net Science Committee, 2012). This function performs weighted averaging. For each user-specified depth slice with upper and lower depth limits $\left(z_{u}, z_{l}\right)$ it finds the $n$ horizons $h_{i}, i \in[1 \ldots n]$ that are contained within or overlap (completely or partially) these limits. These each represent a proportion $p_{i} ; \sum p_{i}=1$ of the thickness $\left(z_{l}-z_{u}\right)$ of the target slab. The attribute values $x_{i}$ (in this case the reflectances) from the $n$ source layers are then combined as weighted averages $\sum_{i} p_{i} \cdot x_{i}$ to give a value for the target slice. These stacked profiles, all with the same horizon limits, were then used as input to linear discriminant analysis (LDA) of the soil orders (highest level in CST), using the lda function of the MASS R package. Prior probabilities for LDA were set to the distribution of the profiles into CST orders. This considers the principal component values at all slices, but not their sequence from top to bottom. Also, because of the standardisation to one meter thickness, the original thickness of the profiles is not represented in the LDA. The distance between LDA centroid means was computed using the dist $\mathrm{R}$ function and converted to similarities as explained in $§ 3.2$.

** Figure 1 about here.

The similarity matrix shown in Figure 1(a) accords with intuition. For example, Aridosols and Halosols are the most similar, while Aridosols are completely dissimilar to Isohumosols. Primosols and Cambosols are also quite similar.

These objectively-computed similarities, based on class centroids in the linear discriminant space, can be directly used as weights in a weighted ac- 
curacy assessment. To illustrate this, we repeated the LDA on a training set of 126 profiles from the set of 189 , selected by stratified random sampling of $2 / 3$ from each order, using the strata function of the sampling $R$ package, and used these class centroids to allocate the remaining 63 evaluation profiles, not used to construct the class centroids, to a class based on the nearest centroid. We also re-computed the distance between LDA centroid means, using only the training set, and used these as the weights matrix for weighted accuracy statistics; these are shown in Figure 1(b). The resulting cross-classification matrix is shown in Table 5 and the naïve and weighted accuracy statistics in Table 6. Overall unweighted accuracy is only 67\%; the largest error is prediction of known Cambosols as Aridosols. Weighting increases the overall accuracy considerably, to $83 \%$, because of the many medium to high similarities in Figure 1, including for the off-diagonals with the most mis-classifications: Aridosols and Cambosols (0.3239) and Aridosols and Halosols (0.7259). The per-class accuracies increase correspondingly.

The $\tau$ statistics are much lower, because of the unbalanced reference class distribution, i.e., mostly Aridosols and Cambosols. The a priori class probabilities were set equal to the reference class distribution in the training set, since these probabilities were used in the discriminant analysis, and these show a high probability of the two dominant classes. Therefore, the weighted $\tau_{w}=0.5337$ even decreases slightly compared to the unweighted $\tau=0.5432$. All these statistics would change somewhat with different training and evaluation sets; the point here is that the weighted statistics give a more realistic view of the value to the user of the use of spectroscopy to allocate unknown profiles to established classes by numerical taxonomy. 


\section{Assessing the accuracy of probabilistic predictions}

The previous sections of this paper have considered the case when a single class is predicted at each location. Some digital soil mapping approaches predict not only the most likely soil class, but also the probability of occurrence of every class in a classification system. This is the approach taken in the SoilGrids project (Hengl et al., 2014), which at the time of the cited paper used multinomial logistic regression to predict the probability of occurrence of each Soil Taxonomy suborder and WRB reference group with one second-level qualifier. In recent versions SoilGrids uses separate binary logistic regressions which are then normalized to sum to one.

The accuracy measures of the previous sections can be used if just the most probable class is taken as the single prediction. However, the multiple predictions allow for more information about map accuracy. The uncertainty of allocation to this most probable class can be measured by Shannon entropy of the probabilities $p_{i}$ for each of the $n$ predicted classes $-\sum_{i=1}^{n} p_{i} \cdot \log _{2}\left(p_{i}\right)$, substituting 0 for $\log _{2}(0)$ for any classes that are not predicted. On the one hand, the most probable class may not be correct, while the correct class is predicted with some probability; the mapping method should get some credit for this. On the other hand, even if the most probable class is correctly predicted, high probabilities of other classes, as shown by high Shannon entropy, should reduce the apparent success of the prediction. This adjustment should also account for class similarity, i.e, taxonomic distance between the several predicted classes. If the classes predicted with the highest probabilities are similar, the reported accuracy should increase to reflect the actual value of the map to the user, and the skill of the mapping method. 
We extend the computations of $\S 4$ to the case where $n$ classes are predicted, each with probability $p_{i}$. Weighted accuracy of a single prediction is then computed as $A=\left(\sum_{i=1}^{n} p_{i} \cdot w_{i a}\right) / \sum_{i=1}^{n} p_{i}$, where $w_{i a}$ is the similarity between predicted class $i$ and the actual class $a ; w_{a a} \equiv 1$. The denominator normalizes the weighted sum, in case of separate predictions. These accuracies can then be summarized either overall or categorized by actual class.

We illustrate this computation with an example of a profile located at geographic coördinates $\left(118.8016^{\circ} \mathrm{E}, 32.13815^{\circ} \mathrm{N}\right)$, collected as part of a project to define soil series, classified as a CST Typic Hapli-Udic Argosol, equivalent to a Soil Taxonomy Udalf. At this location SoilGrids predicts ${ }^{4}$ the probability of occurrence of 67 Soil Taxonomy suborders, of which 31 are non-zero. Shannon entropy is quite high, 4.29, showing the uncertainty of prediction. The first six predictions are Udalfs $(p=0.18)$, Udults $(p=0.16)$, Ochrepts $(p=0.06)$, Udepts, Ustalfs and Ustolls (all $p=0.05$ ); this despite the fact that Ochrepts are no longer a suborder in Soil Taxonomy 2014. Although the most probable class is the correct one, the large uncertainty should be included in the accuracy assessment. To do this, a similarity matrix must be prepared by one of the methods of the previous section. Supposing that the similarity between the Udalfs (correct class) and the other listed classes are $0.8,0.1,0.3,0.2$ and 0 , respectively, the weighted accuracy would be 0.34 , almost double the naïve accuracy 0.18 .

A second example profile from the same project located at geographic coördinates $\left(131.9806^{\circ} \mathrm{E}, 45.65152^{\circ} \mathrm{N}\right)$ is a CST Albic Argi-Udic Isohumosol with a frigid temperature regime, equivalent to a Soil Taxonomy Boroll.

\footnotetext{
${ }^{4}$ https : //rest.soilgrids.org/, 23-Dec-2016
} 
At this location SoilGrids predicts the probability of occurrence of 67 Soil Taxonomy suborders, of which 24 are non-zero. Shannon entropy is again quite high, 4.12. The most probable class is Aquents $(p=0.14)$, which is quite dissimilar from the Borolls. Of the first ten most probable classes only the Borolls (second most probable, $p=0.13$ ), Aquolls (fourth, $p=0.07$ ) and Udolls (tenth, $p=0.04$ ) have any similarity to the correct class. Supposing that the similarity between the Borolls (correct class) and the other two classes with partial credit are 0.2 (Aquolls) and 0.8 (Udolls), the weighted accuracy would be 0.18 , an increase from the naïve accuracy 0.13 , i.e., the probability assigned to the correct class.

\section{Discussion}

We have presented four possible methods to compute taxonomic similarity for the purpose of giving partial credit for misclassifications in a weighted accuracy assessment: expert opinion, distance between classes in a numerical taxonomy assessment, distance within taxonomic hierarchy, and an error loss function.

Of these, the error loss function is the best representation of the effects of a misclassification from the map user's point of view, and can best be converted to a monetary value, and so we prefer it. The difficulty is in formulating the loss function. In the example given above a large amount of tree stand information had to be collected in order to determine the relative yields as site indices. This is only feasible if there is a similar longterm effort, and is especially difficult if the user has multiple objectives. If a land index such as the Storie index (O'Geen et al., 2008) or taxation land value bonitierung system (Rothkegel, 1950) is available and is considered to 
fairly represent the multi-functional value of a soil unit, then these values could be used to determine the relative losses.

The distance in the taxonomic hierarchy is unwieldy and depends on the vagaries of the classification system.

Numerical taxonomy is not as objective as it may first appear, because of choices made in selection of properties and their intercorrelation. These issues have been thoroughly discussed since the pioneering work of Sneath and Sokal (1973), with the conclusion that selecting a large number of properties tends to smooth out any differences, resulting in similar taxonomic distances. In this regard we especially like the use of spectroscopy, which integrates many soil properties in a single spectral signature with thousands of bands. This does not however account for horizon thickness and sequence, and does not include properties destroyed in sampling for spectroscopy, notably soil structure.

Expert opinion is often inconsistent among supposed experts. Especially when evaluating the skill of a soil classifier, the expert opinion of, for example, a regional correlator, is a reasonable way to judge the severity of misallocations. As in any expert judgement, we can guard against extremes by having a panel of experts independently assign partial credits, and then remove the extremes and average the rest. This system is used to judge the skill of gymnasts and divers.

The accuracy measures developed in this paper take the reference class, the so-called ground truth, as correct. It is well-known that some allocation decisions are more difficult than others or more subject to observer bias, also that some allocations depend on measured values close to thresholds used as the so-called taxonomic chop in monothetic classification systems; 
as Butler (1980) pointed out, these introduce taxonomic noise that are the result of human concepts being imposed on natural variation. However, we suppose the user makes decisions based on the predicted class, i.e., consults its description and interpretations. So this assumption of a true reference class does not affect the validity of our procedure.

The methods described in the previous sections apply to cross-classification matrices, where each evaluation observation is allocated to exactly one reference class. Another approach to assessing misallocation in a monothetic system is to blur the boundaries between classes. This approach is known as fuzzy accuracy assessment, and is based on fuzzy allocation of each observation to each class, i.e., each observation has a possibility (not a probability) of being allocated to each reference class. This approach was developed by Gopal and Woodcock (1994) and applied to land cover classification by these authors (Woodcock and Gopal, 2000) and Laba et al. (2002). It requires that each evaluation observation be rated from 1 (allocation completely wrong) to 5 (allocation completely correct) for each possible reference class. If the number of classes is large, the rating of each observation is tedious. For example, even at the most general level of a hierarchical soil classification system (order or reference group), this would require rating each evaluation observation 12 (Soil Taxonomy), 16 (CST), or 14 (WRB) times. For that reason Laba et al. (2002) only rated a 10\% subsample of their 9745 land cover plots representing only 22 of 29 land cover types. However, this method has the advantage that the analysis can report accuracy at various levels of possibility.

This paper could perhaps be considered as evidence in favour of abandoning monothetic soil classification, echoing the lament of Webster (1968). 
Many of the misallocations that are corrected for by partial credit can be attributed to the taxonomic chop of Butler (1980), imposed by the rigid limits of diagnostic properties and horizons, when in fact individuals only differ a small amount from one or more limits. This does not occur in polythetic systems, where classes are based on overall similarity. But as long as studies use monothetic hierarchical systems, we feel that weighted accuracy measures give a more useful estimate of the success of any allocation or mapping method that produces predictions of classes in these systems.

A final point is that decisions which affect the modified accuracy measure, i.e., how properties were selected and the weights matrix computed, should be explicitly stated, i.e., transparent to whoever is judging the map or allocation accuracy. There should be no attempt to manipulate results to make them more favourable. Any positive weighting will raise the unweighted accuracy measures; indeed, giving full partial credit to all misallocations would result in a weighted accuracy of $100 \%$, in which case one might wonder why any allocation was done at all, since all results would be equally useful. Rather, the aim is to report accuracy measures that fairly represent the discrepancy between prediction and reality, according to the needs of the map user or producer. We maintain that if the weighting is fit for the purposes of the evaluation, the weighted measure is a better evaluation of the success of the class predictions.

\section{Acknowledgements}

The first author was supported by an award from the President's International Fellowship Initiative of the Chinese Academy of Sciences. This study was also supported by the National Science Foundation of China 
752 (41130530, 91325301) and Special Project for Basic Data Inventory of the 753 Ministry of Science and Technology of China (2008FY110600). We thank

Professor De-Cheng Li for his expert opinion on the similarity between the soil classes in the first example of $\S 5$.

\section{References}

Avery, T. E., Burkhart, H. E., 2015. Forest measurements, 5th Edition. Waveland Press, Long Grove, IL.

Beaudette, D. E., Roudier, P., O'Geen, A. T., 2013. Algorithms for quantitative pedology: A toolkit for soil scientists. Computers \& Geosciences 52, 258-268.

Bidwell, O., Hole, F., 1964. An experiment in the numerical classification of some Kansas soils. Soil Science Society of America Proceedings 28, 263-268.

Brus, D., Kempen, B., Heuvelink, G., 2011. Sampling for validation of digital soil maps. European Journal of Soil Science 62, 394-407.

Buol, S. W., Southard, R. J., Graham, R. C., McDaniel, P., 2011. Soil genesis and classification. Wiley-Blackwell, Chichester, UK.

Butler, B., 1980. Soil classification for soil survey. Clarendon Press, Oxford.

Carré, F., Jacobson, M., 2009. Numerical classification of soil profile data using distance metrics. Geoderma 148, 336-345.

Cohen, J., 1960. A coefficient of agreement for nominal scales. Educational \& Psychological Measurement 20, 37-46.

Congalton, R. G., Green, K., 1999. Assessing the accuracy of remotely sensed data: principles and practices. Lewis, Boca Raton, FL.

Cooperative Research Group on Chinese Soil Taxonomy, 2001. Chinese Soil Taxonomy. Science Press, Beijing.

de Gruijter, J., 1977. Numerical classification of soils and its application in survey. Vol. 855 of Agricultural Research Report. PUDOC, Wageningen (NL).

Dobos, E., Vadnai, P., Bertoti, D., Kovacs, K., Micheli, E., Lang, V., Fuchs, M., 2014. A novel approach for validating raster datasets with categorical data. In: GlobalSoilMap: 
Basis of the global spatial soil information system. CRC Press-Taylor \& Francis Group, pp. 347-351, Boca Raton, FL.

Driessen, P., Deckers, J., Spaargaren, O., Nachtergaele, F., 2001. Lecture notes on the major soils of the world: World Soil Resources Reports 94. World Soil Resources Reports. FAO, Rome.

Edmonds, W. J., Lentner, M., 1986. Statistical evaluation of the taxonomic composition of three soil map units in Virginia. Soil Science Society of America Journal 50, 9971001.

FAO, 2014. World Reference Base for Soil Resources 2014: International soil classification system for naming soils and creating legends for soil maps. No. 106 in World Soil Resources Reports. Food and Agriculture Organization of the United Nations, Rome. URL http://www.fao.org/3/a-i3794e.pdf

Finke, P. A., 2007. Quality assessment of digital soil maps: producers and users perspectives. In: Digital Soil Mapping: An Introductory Perspective. Elsevier Scientific, Ch. 31, pp. 523-541, Amsterdam.

Foody, G. M., 1992. On the compensation for chance agreement in image classification and accuracy assessment. Photogrammetric Engineering \& Remote Sensing 58, 14591460.

Gerasimova, M. I., 2010. Chinese Soil Taxonomy: Between the American and the international classification systems. Eurasian Soil Science 43, 945-949.

GlobalSoilMap.net Science Committee, 2012. Specifications: Tiered GlobalSoilMap.net products; Release 2.3. Federation University Australia, Ballarat, Victoria (Australia). URL http://www.ozdsm.com.au/resources/GlobalSoilMap\%20specs\%20version\% 202point3.pdf

Gopal, S., Woodcock, C., 1994. Theory and methods for accuracy assessment of thematic maps using fuzzy sets. Photogrammetric Engineering \& Remote Sensing 60, 181-188.

Gower, J. C., 1971. A general coefficient of similarity and some of its properties. Biometrics $27,857-871$.

Hengl, T., de Jesus, J. M., MacMillan, R. A., Batjes, N. H., Heuvelink, G. B. M., Ribeiro, E., Samuel-Rosa, A., Kempen, B., Leenaars, J. G. B., Walsh, M. G., et al., 2014. SoilGrids1km - Global soil information based on automated mapping. PLoS 
ONE 9, e105992.

Laba, M., Gregory, S. K., Braden, J., Ogurcak, D., Hill, E., Fegraus, E., Fiore, J., DeGloria, S. D., 2002. Conventional and fuzzy accuracy assessment of the New York Gap Analysis Project land cover map. Remote Sensing of Environment 81, 443-455.

Láng, V., Fuchs, M., Szegi, T., Csorba, A., Michéli, E., 2016. Deriving World Reference Base Reference soil groups from the prospective Global Soil Map product - a case study on major soil types of Africa. Geoderma 263, 226-233.

Láng, V., Fuchs, M., Waltner, I., Michéli, E., 2013. Soil taxonomic distance, a tool for correlation: As exemplified by the Hungarian Brown Forest Soils and related WRB Reference Soil Groups. Geoderma 192, 269-276.

Leenaars, J. G. B., 2013. Africa Soil Profiles Database, Version 1.1. A compilation of geo-referenced and standardized legacy soil profile data for Sub Saharan Africa (with dataset). ISRIC-World Soil Information, ISRIC Report 2013/03, Wageningen, NL.

Ma, Z., Redmond, R. L., 1995. Tau coefficients for accuracy assessment of classification of remote sensing data. Photogrammetric Engineering \& Remote Sensing 61 (4), 435439.

McBratney, A., Minasny, B., 2007. On measuring pedodiversity. Geoderma 141, 149-154.

McBratney, A. B., Mendonça Santos, M. L., Minasny, B., 2003. On digital soil mapping. Geoderma 117, 3-52.

Michéli, E., Láng, V., Owens, P. R., McBratney, A., Hempel, J., 2016. Testing the pedometric evaluation of taxonomic units on Soil Taxonomy - A step in advancing towards a universal soil classification system. Geoderma 264, 340-349.

Minasny, B., McBratney, A. B., Hartemink, A. E., 2010. Global pedodiversity, taxonomic distance, and the World Reference Base. Geoderma 155, 132-139.

Moore, A. W., Russell, J. S., Ward, W. T., 1972. Numerical analysis of soils: a comparison of three soil profile models with field classification. Journal of Soil Science 23, 193-209.

Odgers, N. P., McBratney, A. B., Minasny, B., 2011. Bottom-up digital soil mapping. II. Soil series classes. Geoderma 163, 30-37.

O'Geen, A. T., Southard, S. B., Southard, R. J., 2008. A revised Storie index for use with digital soils information. No. 8335 in University of California Division of Agriculture and Natural Resources. University of California Division of Agriculture and Natural 
Resources, Davis, CA (USA).

URL http://anrcatalog.ucdavis .edu/pdf/8335.pdf

Oreskes, N., Shrader-Frechette, K., Belitz, K., 1994. Verification, validation, and confirmation of numerical models in the earth sciences. Science 263, 641-646.

Phillips, J. D., 2013. Evaluating taxonomic adjacency as a source of soil map uncertainty. European Journal of Soil Science 64, 391-400.

Rossiter, D. G., 1995. Economic land evaluation: why and how. Soil Use \& Management $11,132-140$.

Rothkegel, W., 1950. Geschichtliche Entwicklung der Bodenbonitierungen, und Wesen und Bedeutung der deutschen Bodenschätzung. E. Ulmer, Stuttgart.

Scull, P., Franklin, J., Chadwick, O., McArthur, D., 2003. Predictive soil mapping: a review. Progress in Physical Geography 27, 171-197.

Smith, G., 1986. The Guy Smith interviews: rationale for concepts in Soil Taxonomy. SMSS Technical Monograph 11. Soil Management Support Service, Washington, DC. URL http://www.nrcs.usda.gov/wps/portal/nrcs/detail/soils/survey/ class/taxonomy/?cid=nrcs142p2_053582

Sneath, P. H. A., 1995. Thirty years of numerical taxonomy. Systematic Biology 44, 281-298.

Sneath, P. H. A., Sokal, R. R., 1973. Numerical taxonomy: the principles and practice of numerical classification. San Francisco, US: Freeman.

Soil Survey Staff, 2014. Keys to Soil Taxonomy, 12th Edition. US Government Printing Office, Washington, DC.

Stehman, S. V., Czaplewski, R. L., 1998. Design and analysis for thematic map accuracy assessment: Fundamental principles. Remote Sensing of Environment 64, 331-344.

Taghizadeh-Mehrjardi, R., Nabiollahi, K., Minasny, B., Triantafilis, J., Sep 2015. Comparing data mining classifiers to predict spatial distribution of USDA-family soil groups in Baneh region, Iran. Geoderma 253, 67-77.

USDA-NRCS, 2013. Web Soil Survey. Accessed 03-August-2016.

URL http://websoilsurvey.sc.egov.usda.gov

USDA-NRCS, N.D. USDA-NRCS Official Soil Series Descriptions and series classification. Accessed 03-August-2016. 
URL https://soilseries.sc.egov.usda.gov/

van Beek, L., 2014. Statistical validation of soil type maps for Africa. No. SGL-80812 WUR in BSc thesis Soil, Water, Atmosphere. Wageningen University Research, Wageningen $(\mathrm{NL})$.

van Huyssteen, C. W., Micheli, E., Fuchs, M., Waltner, I., 2014. Taxonomic distance between South African diagnostic horizons and the World Reference Base diagnostics. Catena 113, 276-280.

Van Wambeke, A., Nachtergaele, F., 2003. Properties and management of soils of the tropics. FAO Land and Water Digital Media Series. FAO, Rome.

Vepraskas, M. J., 2015. History of the concept of hydric soil. In: Wetland soils: genesis, hydrology, landscapes, and classification, 2nd Edition. CRC Press, Ch. 2, pp. 23-38.

Viscarra Rossel, R. A., Webster, R., 2011. Discrimination of Australian soil horizons and classes from their visible-near infrared spectra. European Journal of Soil Science 62, $637-647$.

Webster, R., 1968. Fundamental objections to the 7th approximation. Journal of Soil Science 19, 354-366.

Woodcock, C., Gopal, S., 2000. Fuzzy set theory and thematic maps: Accuracy assessment and area estimation. International Journal of Geographical Information Science $14,153-172$.

Young, F. J., Hammer, R. D., 2000. Defining geographic soil bodies by landscape position, soil taxonomy, and cluster analysis. Soil Science Society of America Journal 64, 989998.

Zeng, R., Zhang, G.-L., Li, D.-C., Rossiter, D. G., Zhao, Y.-G., 2016. How well can VNIR spectroscopy distinguish soil classes? Biosystems Engineering 152, 117-125. 


\begin{tabular}{|llr|}
\hline Series & Soil Taxonomy family & $\begin{array}{r}\text { Site } \\
\text { index }\end{array}$ \\
\hline Rains & Fine-loamy, siliceous, semiactive, thermic Typic Paleaquults & 94 \\
Lynchburg & Fine-loamy, siliceous, semiactive, thermic Aeric Paleaquults & 86 \\
Goldsboro & Fine-loamy, siliceous, subactive, thermic Aquic Paleudults & 88 \\
Norfolk & Fine-loamy, kaolinitic, thermic Typic Kandiudults & 84 \\
Wagram & Loamy, kaolinitic, thermic Arenic Kandiudults & 81 \\
\hline Tarboro & Mixed, thermic Typic Udipsamments & 72 \\
\hline
\end{tabular}

Table 1: Some North Carolina soil series and their site indices for Pinus taeda 


\begin{tabular}{|l|rrrrrr|}
\hline Suborder & OP & SA & UA & UC & AV & AC \\
\hline OP & $\mathbf{2}$ & 1 & 0 & 5 & 0 & 0 \\
SA & 1 & $\mathbf{7 4}$ & 2 & 1 & 3 & 6 \\
UA & 0 & 5 & $\mathbf{8}$ & 6 & 1 & 3 \\
UC & 6 & 1 & 3 & $\mathbf{9 1}$ & 0 & 0 \\
AV & 0 & 4 & 0 & 0 & $\mathbf{0}$ & 4 \\
AC & 0 & 6 & 2 & 2 & 4 & $\mathbf{3 8}$ \\
\hline
\end{tabular}

Table 2: Confusion matrix of predictions for suborder level $\mathrm{OP}=$ Orthic Primosols; $\mathrm{SA}=$ Stagnic Argosols; UA = Udic Argosols; UC = Udic Cambosols; $\mathrm{AV}=$ Aquic Vertosols; $\mathrm{AC}=$ Aquic Cambosols Rows: as allocated; columns: reference class. 


\begin{tabular}{|l|rrrrrr|}
\hline Suborder & OP & SA & UA & UC & AV & AC \\
\hline OP & $\mathbf{1}$ & 0.05 & 0.05 & 0.15 & 0.05 & 0.15 \\
SA & 0.05 & $\mathbf{1}$ & 0.05 & 0.05 & 0.05 & 0.35 \\
UA & 0.05 & 0.05 & $\mathbf{1}$ & 0.20 & 0.15 & 0.15 \\
UC & 0.15 & 0.05 & 0.25 & $\mathbf{1}$ & 0.10 & 0.25 \\
AV & 0.05 & 0.10 & 0.15 & 0.10 & $\mathbf{1}$ & 0.15 \\
AC & 0.20 & 0.30 & 0.10 & 0.25 & 0.20 & $\mathbf{1}$ \\
\hline
\end{tabular}

Table 3: Weights matrix based on expert opinion

$\mathrm{OP}=$ Orthic Primosols; $\mathrm{SA}=$ Stagnic Argosols; UA = Udic Argosols; UC = Udic Cambosols; $\mathrm{AV}=$ Aquic Vertosols; $\mathrm{AC}=$ Aquic Cambosols

Rows: as allocated; columns: reference class. 


\begin{tabular}{|c|c|c|c|c|}
\hline \multicolumn{4}{|c|}{ Unweighted accuracy $A_{o}$ : } & \\
\hline \multicolumn{4}{|c|}{ Weighted accuracy $A_{o w}$ : } & 0.8039 \\
\hline & \multicolumn{3}{|c|}{ Unweighted tau $\tau$ : } & 0.7161 \\
\hline & \multicolumn{3}{|c|}{ Weighted tau $\tau_{w}$ : } & 0.7253 \\
\hline & UA & $\mathrm{UA}(\mathrm{W})$ & $\mathrm{PA}$ & $\mathrm{PA}(\mathrm{W})$ \\
\hline OP & 0.25 & 0.35 & 0.22 & 0.33 \\
\hline SA & 0.85 & 0.88 & 0.81 & 0.84 \\
\hline UA & 0.35 & 0.44 & 0.53 & 0.60 \\
\hline UC & 0.90 & 0.92 & 0.87 & 0.89 \\
\hline $\mathrm{AV}$ & 0.00 & 0.12 & 0.00 & 0.14 \\
\hline $\mathrm{AC}$ & 0.73 & 0.79 & 0.75 & 0.81 \\
\hline
\end{tabular}

Table 4: Accuracy statistics based on expert weighting; equal prior probabilities Per class: UA, PA = User's, Producer's accuracy; $(\mathrm{W})=$ weighted 


\begin{tabular}{|l|rrrrrr|}
\hline Order & Anthrosols & Aridosols & Cambosols & Halosols & Isohumosols & Primosols \\
\hline Anthrosols & $\mathbf{2}$ & 0 & 1 & 0 & 0 & 0 \\
Aridosols & 1 & $\mathbf{1 4}$ & 5 & 3 & 0 & 3 \\
Cambosols & 0 & 2 & $\mathbf{1 7}$ & 0 & 1 & 1 \\
Halosols & 0 & 0 & 0 & $\mathbf{2}$ & 0 & 2 \\
Isohumosols & 0 & 0 & 1 & 0 & $\mathbf{1}$ & 0 \\
Primosols & 0 & 1 & 0 & 0 & 0 & $\mathbf{6}$ \\
\hline
\end{tabular}

Table 5: Cross-classification matrix, evaluation of linear discriminant analysis of 126 training spectra on 63 evaluation profiles.

Rows: as allocated; columns: reference class. 


\begin{tabular}{rrrrr} 
Unweighted accuracy $A_{o}:$ & 0.6667 \\
Weighted accuracy $A_{o w}:$ & 0.8269 \\
Unweighted tau $\tau:$ & 0.5432 \\
Weighted tau & $\tau_{w}:$ & 0.5337 \\
\hline & $\mathrm{UA}$ & $\mathrm{UA}(\mathrm{W})$ & $\mathrm{PA}$ & $\mathrm{PA}(\mathrm{W})$ \\
\hline Anthrosols & 0.67 & 0.83 & 0.67 & 0.87 \\
Aridosols & 0.54 & 0.76 & 0.82 & 0.89 \\
Cambosols & 0.81 & 0.91 & 0.71 & 0.83 \\
Halosols & 0.50 & 0.73 & 0.40 & 0.83 \\
Isohumosols & 0.50 & 0.79 & 0.50 & 0.79 \\
Primosols & 0.86 & 0.91 & 0.50 & 0.73
\end{tabular}

Prior probabilities for $\tau$ from training set: Anthrosols 0.0322; Aridosols 0.2903;

Cambosols 0.3871; Halosols 0.0645; Isohumosols 0.0326; Primosols 0.1935

Table 6: Accuracy statistics based on spectral similarity according of Figure 1 
Figures

\section{List of Figures}

910 1 Taxonomic similarity between class centroids determined by

$911 \quad$ linear discriminant analysis on spectra, based on (a) all 189

912

profiles; (b) training set of 126 profiles. . . . . . . . . . . . 48 


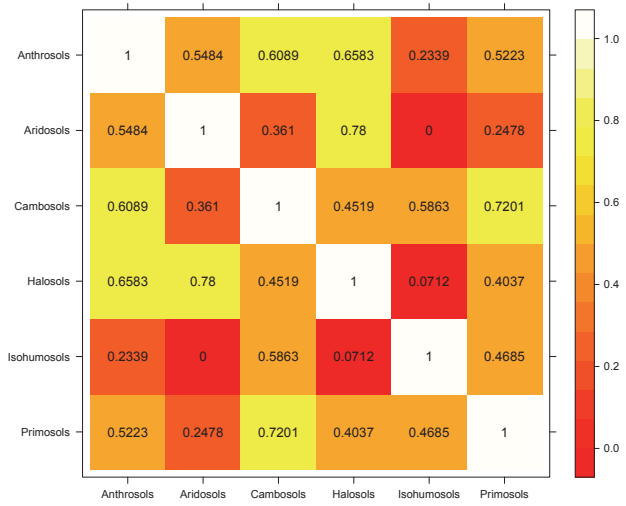

(a)

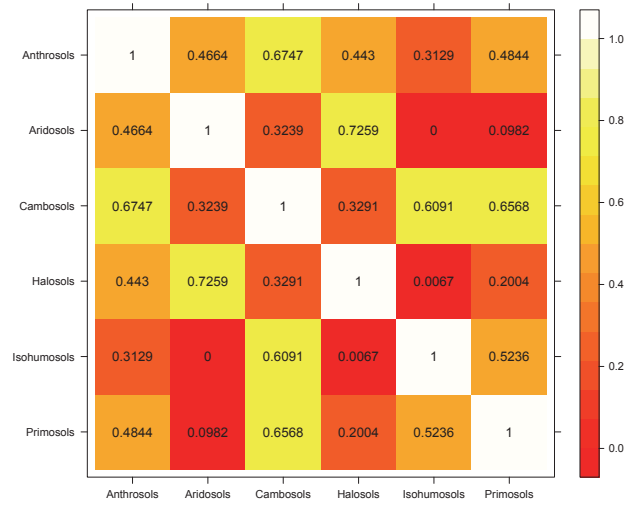

(b)

Figure 1: Taxonomic similarity between class centroids determined by linear discriminant analysis on spectra, based on (a) all 189 profiles; (b) training set of 126 profiles. 